\title{
ENDOVASCULAR TREATMENT IN ACUTE ISCHEMIC STROKE
}

\author{
Silva Andonova ${ }^{1}, Z^{2}$ etomila Dimitrova ${ }^{1}$, Evgenia Kalevska ${ }^{1}$, Marina Petkova ${ }^{1}$, \\ Vania Argirova ${ }^{1}$, Penka Kirilova ${ }^{1}$, Zvetan Zvetkov ${ }^{1}$, Marianna Novakova ${ }^{2}$, \\ Radoslav Georgiev ${ }^{2}$ \\ ${ }^{1}$ Department of Neurology, Medical University of Varna, ${ }^{2}$ Centre of Radiology, \\ St. Marina University Hospital of Varna
}

\begin{abstract}
Stroke remains a major health care challenge worldwide and is the third leading cause of death and the leading cause of disability in developed countries. The management of acute ischemic stroke has advanced greatly over the past 20 years. Now the treatment of stroke can be described with one word- variety.

The current review is dedicated to the problems of endovascular treatment in acute ischemic stroke. The different methods of treatment, pros and cons of each of them, discussing the different options for the patients are presented.
\end{abstract}

Keywords: intravenous thrombolysis, intra-arterial thrombolysis, mechanical clot removal, stent placement

Every stroke centre worked based on some kind of protocols or administrative pathways, but at the moment there do not exist united positions for a lot of questions, related to stroke management. The main goal in treatment is to protect the penumbra (this is the area surrounding an ischemic event such as an ischemic, thrombotic or embolic stroke) by increasing oxygen transport and delivery to cells in the danger zone, thereby limiting cell death. The existence of a penumbra implies that salvaging of the

\footnotetext{
Address for correspondence:

Silva Andonova, $M D P h D$

Department of Neurology,

Head of Second Clinic of Neurology

University Hospital "St. Marina"

1 HristoSmirnenski Str

9010 Varna, Bulgaria

Tel: +35952978236

e-mail:drsilva@abv.bg
}

Received: November 20, 2013

Accepted: February 11, 2014 cells is possible. There is a high correlation between the extent of spontaneous neurological recovery and the volume of penumbra that escapes infarction. Therefore, saving the penumbra should improve the clinical outcome. Different studies are going at the moment, trying to determine the best way to save this fragile area and treat a patient with ischemic stroke. Although there are a lot of different opinions, also there exists some golden standards, which are applied in major stroke centers, such as intravenous (IV) thrombolysis.

The era of endovascular therapy for acute ischemic stroke began with the first reported use of thrombolytic agents. Since the approval in 1996 by the United States Food and Drug Administration (FDA) of intravenous thrombolysis with recombinant tissue plasminogen activator (rtPA, alteplase) for the treatment of acute ischemic stroke is the standard treatment $(3,6)$. Intravenous rt-PA in acute ischemic stroke should be undertaken in patients satisfying specific inclusion and exclusion criteria. It should be given as early as possible, because the effect size of 
Silva Andonova, Zvetomila Dimitrova, Evgenia Kalevska et al.

thrombolysis is time-dependent. Where it is possible, therapy should commence in the first few hours but may be used up to 4,5 hours after stroke onset. These procedures required appropriate hospital infrastructure, facilities and network support. Also IV rt-PA should be given under the authority of a physician trained and experienced in acute stroke management. For as much as there are specific criteria or protocol, IV thrombolysis cannot be performed in every patient with acute ischemic stroke. One of the main limitations to IV rtPA since its approval has been the strict at first 3-hour then 4.5- hour time window for initiating therapy. This window, combined with a lack of public awareness of stroke, has unfortunately limited the use of IV thrombolysis to $<5 \%$ of eligible candidates. So if the time window is passed, there are other possibilities for treatment such as IA trombolysis and other endovascular methods.

Intra-arterial (IA) thrombolysis is an emerging treatment strategy for acute ischemic stroke. It was first described in 1983 by Zeumer et al. One hundred eighty patients with proximal middle cerebral artery (MCA) occlusions were enrolled and treated within $6 \mathrm{hr}$ of the onset of stroke. Additional inclusion criteria included a National Institutes of Health Stroke Scale score of 4 to 30 and lack of haemorrhage or early signs of infarction involving more than onethird of the MCA territory on CT scans. Exclusion criteria included rapidly improving neurologic deficit or sustained blood pressure of $>180 / 100$. In IA thrombolysis, the cervicocephalic arterial tree is traversed with an endovascular microcatheter delivery system, the catheter port is positioned immediately within and adjacent to the offending thrombus, and fibrinolytic agents are infused directly into the clot. This delivery technique permits high concentrations of lytic agent to be applied to the clot while minimising systemic exposure. MRI studies have provided striking imaging evidence of the potential beneficial effects of IA therapy $(12,15)$. As with other interventional techniques used in acute stroke, the major disadvantages to IAT include the relative complexity of the procedure, the level of required technical expertise, relatively selective availability, delays in initiating treatment, and the additional risks and expense of an invasive procedure compared with IVT. The risk of symptomatic intracerebral haemorrhage and limited time constraints associated with thrombolytic agents prompted a search for alternate means of recanalisation in acute ischemic stroke. In some cases, where the patient has excluding factors and it is impossible to perform IV or IA thrombolysis, or administrating the latter doesn't show any result, we can use alternative treatment (18).

There is still insufficient evidence to recommend the use of mechanical clot removal in routine clinical practice, but in coming years endovascular mechanical reperfusion techniques, is likely to be increasingly refined and validated and to become a widely accepted therapy for acute ischemic stroke. The MERCI (The Mechanical Embolus Removal in Cerebral Ischemia) retriever was the first stroke device to be approved by the FDA, and thus launched the era of mechanical thrombectomy. „To restore blood flow in the neurovasculature by removing thrombus in patients experiencing ischemic stroke. Patients who are ineligible for treatment with IVrtPA or who fail IV-rtPA therapy are candidates for treatment" $(5,8)$. The Penumbra system is the second (and so far, the last) FDA-approved device specifically developed for the purpose of clot removal in acute ischemic stroke $(9,10)$. This system provides a dual approach to clot extraction using aspiration and debulking of the thrombus to reduce or eliminate the clot burden. This is followed by clot retrieval with a ring device if needed that engages the thrombus by capturing it in clasps with a cylinder that is then withdrawn during flow arrest. Now there are a lot of studies and compartments between the intravenous thrombolysis and mechanical device and intraarterial t-PA. But a lot of the questions are still unanswered, the time and the indications for each of the procedures are vague.

On the other hand, it still remains the question of time management. For example, performing a carotid endarterectomy or stent placements in patients with ischemic stroke during the acute phaze. It is considered that the time between the second day and the second week is the most suitable for performing a carotid endarterectomy. 
Carotid endarterectomy is still the golden standard for treatment of the carotid artery stenosis. But many studies have shown the feasibility and high efficacy of percutaneous transluminal angioplasty (PTA) in acute stroke $(1,3)$.

The angioplasty and stent placement are most commonly used in patients with acute ischemic stroke and desiccation of carotid artery. Jovin et al achieved successful recanalisation in 23 of 25 patients, which had emergency stent placement on the extracranial internal carotid artery. Brekenfeld et al treated 350 patients with intraarterial urokinase and noticed that recanalisation can be improved by angioplasty and stent implantation $(2,3)$.

One of the most exciting recent developments in the interventional treatment of acute ischemic stroke was perhaps the use of self-expanding stents for flow restoration. The first prospective FDA-approved trial, Stent-Assisted Recanalization in Acute Ischemic Stroke, demonstrated $100 \%$ recanalisation rate in 20 patients. At present, no time recommendation for initiation of endovascular treatment in acute ischemic stroke is available. A recent multicentre analysis was designed to identify variables that prolong "time to microcatheter," defined as the time interval from computed tomographic scan to microcatheter placement in the cerebral circulation $(5,13,17)$.

\section{CONCLUSION}

Nowadays the treatment of ischemic stroke has more options than ever. The possibility of catheter-based drug administration, mechanical embolectomy, and angioplasty with stent placement required strict indication and unified protocols. The time and the indications for each of the procedures are questions, without precise answers.

\section{REFERENCES}

1. Balwinder S., P. Ajay, P. Larry, M. Manoj, Endovascular Therapy for Acute Ischemic Stroke: A Systematic Review and Meta-analysis. Mayo Clinic Proceedings, 2013;88(10):1056-1065.

2. Bambauer K.Z., S.C. Johnston, D.E. Bambauer, J.A. Zivin. Reasons why few patients with acute stroke receive tissue plasminogen activator. Arch Neurol, 63, 2006, No 5, 661-664;

3. Barber P.A., J. Zhang, A.M. Demchuk, M.D. Hill, A.M. Buchan. Why are stroke patients excluded from TPA therapy? An analysis of patient eligibility. Neurology, 56, 2001, No 8, 1015-1020;

4. Blackham K.A., P.M. Meyers, T.A. Abruzzo, F.C. Alberquerque, D. Fiorella, J. Fraser, D. Frei. Endovascular Therapy of Acute Ischemic Stroke. J NeuroIntervent Surg, 4, 2012; No 2, 87-93.

5. Broderick J.P. Endovascular therapy for acute ischemic stroke. Stroke, 40, 2009, No 7, 103-106.

6. Broderick J.P., Y.Y. Palesch, A.M. Demchuk. Endovascular therapy after intravenous t-PA versus t-PA alone for stroke. N Engl J Med. 368, 2013, No 10, 893-903;

7. Broussalis E, E. Trinka, W. Hitzl, A. Wallner, V. Chroust, M. Killer-Oberpfalzer . Comparison of stent-retriever devices versus the Merci retriever for endovascular treatment of acute stroke. Am J Neuroradiol., 34, 2013, No 2, 366-372;

8. Ciccone A, L. Valvassori, M. Nichelatti, A. Sgoifo, M. Ponzio, R. Sterzi ; Endovascular treatment for acute ischemic stroke. N Engl J Med., 368, 2013, No 10, 904-913.

9. Chimowitz M.I. Endovascular treatment for acute ischemic stroke-still unproven. N Engl J Med., 368, 2013, No 10, 952-955;

10. Hacke W, H. Zeumer, A. Ferbert, H. Brückmann, G.J. del Zoppo. Intra-arterial thrombolytic therapy improves outcome in patients with acute vertebrobasilar occlusive disease. Stroke, 19, 1988, No 10, 1216-1222;

11. Hacke W., M. Kaste, E. Bluhmki. Thrombolysis with alteplase 3 to 4.5 hours after acute ischemic stroke. N Engl J Med, 359, 2008, No 13, 1317-1329;

12. Mori E, M. Tabuchi, T. Yoshida, A. Yamadori. Intracarotid urokinase with thromboembolic occlusion of the middle cerebral artery. Stroke, 19, 1988, No 7, 802-812;

13. Nam J., H. Jing, D. O'Reilly. Intra-arterial thrombolysis vs. standard treatment or intravenous thrombolysis in adults with acute 
ischemic stroke: a systematic review and metaanalysis. Int J Stroke, 12, 2013, No 7, 227-312.

14. Nogueira R.G., L.H. Schwamm, J.A. Hirsch. Endovascular approaches to acute stroke, part 1: drugs, devices, and data. AJNR Am J Neuroradiol, 30, 2009, No 4, 649-661;

15. Rha J.H., J.L. Saver . The impact of recanalization on ischemic stroke outcome: a meta-analysis. Stroke, 38, 2007, No 3, 967-973;

16. Theron J., P. Courtheoux, A. Casasco. Local intraarterial fibrinolysis in the carotid territory. AJNR Am J Neuroradiol, 10, 1989, No 4, 753-765;;

17. Qureshi A.I., A.M. Siddiqui, M.F. Suri. Aggressive mechanical clot disruption and low-dose intra-arterial thirdgeneration thrombolytic agent for ischemic stroke: a prospective study. Neurosurgery, 51, 2002, No 5, 1319-1327;

18. Zeumer H., H.J. Freitag, U. H.P. Grzyska, Neunzig. Local intraarterial fibrinolysis in acute vertebrobasilar occlusion. Technical developments and recent results. Neuroradiology, 31, 1989, No 4, 336-340. 